\title{
Evaluation and management of gender identity disorder -an American tale
}

T he Gender Management Service (GeMS) Clinic opened in 2007 as an extension to services already offered at Children's Hospital Boston. It is the first focused, multidisciplinary group in North America offering medical treatment to gender-dysphoric children. Using methods largely developed in The Netherlands (the so-called Dutch Protocol), the clinicians and researchers recently reported their experiences in Pediatrics, advocating for the early evaluation of these children and for consideration of their stage of puberty rather than their age.

Guidelines from the Endocrine Society on the treatment of transsexual patients state that prepubertal children should not undergo gender-changing therapy. Instead, young patients (Tanner stage 2 or 3) should undergo puberty blockade with gonadotrophin-releasing hormone analogues (GnRHa). The reasoning for this guideline is the high rate of remission of gender identity disorder (GID) after the onset of puberty. "Adolescents with an unstable, and possibly unsettled, gender role and identity can, and occasionally do, change their minds, sometimes more than once," explains expert endocrinologist David Handelsman of the ANZAC Research Institute at The University of Sydney, Australia, who is not involved with the GeMS clinic. "Puberty suppression with GnRHa is the best way to support adolescent patients in their desires to interrupt the unwanted features of adult sex as well as deferring definitive treatment, including surgery, until the patient has attained greater personal maturity."

The team at the GeMS Clinic, led by Norman Spack and David Diamond, used the time gained from GnRHa treatment for counseling and psychotherapy, and to give their young patients the opportunity to explore being the new gender before deciding to irreversibly change his or her body. The extra time also gives patients and their families the opportunity to

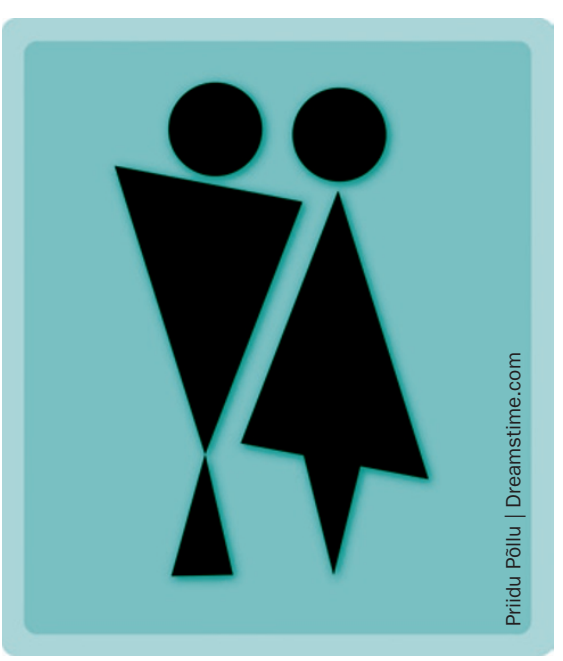

consider the costs and benefits of any long-term change. Also, they did not restrict puberty-cessation therapy to children older than 12 years, as the Amsterdam Gender Clinic did. Instead, patients who had reached Tanner stage 2 were eligible for puberty-cessation therapy.

After the clinic opened, the number of patients presenting at Children's Hospital Boston increased fourfold indicating an improved awareness of GID by the general public. However, the majority of young people who did seek help were already pubescent: with a mean Tanner stage of $4.1 \pm 1.4$ for biologically female patients and $3.6 \pm 1.5$ for biologically male patients. Of the 97 patients ( 54 biologically female, 43 biologically male) the team treated since 1998 , only $20 \%$ were at Tanner stage 2 or 3 and received GnRHa treatment; $11 \%$ were not yet Tanner stage 2 and had to wait for treatment. By contrast, $69 \%$ of patients presented at Tanner stage 4 or 5 ; the likelihood that irreversible secondary sexual characteristics have already formed in these patients is higher, as is the likelihood they will require extensive surgical procedures-such as mammoplasty-should they decide to undergo gender transformation. Limited access to care might have a role in explaining this delay in help-seeking.
Patients with GID at advanced Tanner stage are more difficult, and more costly, to treat. GnRHa therapy at advanced Tanner stages can reach upwards of $\$ 1,000$ per month (out of pocket expense), plus the cost of the necessary co-treatment with cross-sex hormones. Financial constraints can mean that patients do not get the care they need. "In the USA, key decision making on optimal treatment is being dominated by access to care (affordability), something that is not a factor, let alone a decisive factor, in countries with effective national health schemes like Holland," Handelsman comments.

As well as cost, many patients with GID can be burdened by psychiatric symptoms. Indeed, a striking majority of these young people are at increased risk of self-harming, abusing illicit substances and attempting suicide. All patients at the GeMS Clinic-44\% of whom had a prior history of psychiatric diagnosis-underwent comprehensive psychometric testing, which examined features including depression, anxiety, gender dysphoria scale and body image. In another study, also new in Pediatrics, Roberts et al. identified that transgender youths were at increased risk of abuse (sexual, physical and psychological) and post-traumatic stress disorder, which complicates the situation further. The experience of the GeMS team underpins the value of specialized, mutlidisciplinary teams dedicated to identifying the signs of gender dysphoria and treating not only the physical symptoms but also the psychological consequences.

\section{Mina Razzak}

